Original Paper http://ajol.info/index.php/ijbcs http://indexmedicus.afro.who.int

\title{
Revenus économiques et pollution écosystémique liés au transport des personnes et des biens traversant les mangroves de l'estuaire du Wouri (Douala, Cameroun)
}

\author{
Gordon Nwutih AJONINA ${ }^{2,3}$, Siegfried Didier DIBONG ${ }^{1}$, Rodrigue EBONJI SETH ${ }^{2 *}$, \\ Yevalla GAH-MUTI², Nathalie ELOUGOU NDINGA ${ }^{2}$ et Alexia NKOMBA ${ }^{2}$ \\ ${ }^{I}$ Faculté des Sciences de l'Université de Douala, Cameroun. \\ ${ }^{2}$ Institut des Sciences Halieutiques de l'Université de Douala à Yabassi, Cameroun. \\ ${ }^{3}$ Cameroon Wildlife Conservation Society (CWCS) Mouanko, Cameroun. \\ *Auteur correspondant, E-mail : ebonjiseth@yahoo.fr
}

\section{RESUME}

La mangrove est une formation forestière caractéristique des littoraux tropicaux vaseux, essentiellement constituée de palétuviers. Au Cameroun, les mangroves couvrent une superficie de $2700 \mathrm{~km}^{2}$, soit $1,5 \%$ de la superficie des forêts, répartie telle que $1600 \mathrm{~km}^{2}$ occupent le Rio Del Rey et $1100 \mathrm{~km}^{2}$ l'Estuaire du Cameroun. Une étude sur les paiements écosystèmiques a été conduite dans l'écosystème mangrove de l'estuaire du Wouri pour évaluer les enjeux financiers et les risques de pollution liés au transport des personnes et des marchandises. La méthode d'échantillonnage s'est faite aléatoirement sur 27 pirogues dans deux débarcadères principaux de mangroves dans la localité, dont 20 à Youpwè et 07 à Akwa-Nord, avec les observations basées sur l'utilisation des fiches d'enquêtes socio-économiques. Les résultats obtenus montrent que les services écosystèmiques constituent un moyen de subsistence pour les populations riveraines. Le flux des personnes et des marchandises est important et équivaut à un chiffre d'affaires de $12252600 \mathrm{~F}$ CFA (24 505 USD)/pirogue/an à Youpwè et 3896286 F CFA (7 792 USD)/pirogue/an à Akwa-Nord. Toutefois, les activités anthropiques liées à ce transport constituent des menaces pour sa pérennisation.

(C) 2015 International Formulae Group. All rights reserved.

Mots clés : Mangrove, transport, services écosystemiques, enjeux financiers, menaces.

\section{Economic incomes and ecosystemic pollution related to the transportation of persons and goods through the mangroves of the Wouri estuary (Douala, Cameroon)}

\section{ABSTRACT}

The mangrove is a forest formation characteristic of muddy tropical coastlines, mostly constituted of mangroves. In Cameroon, mangroves covers a superficial area of $2700 \mathrm{~km}^{2}$, about $1.5 \%$ of the superficial area, divided into $1600 \mathrm{~km}^{2}$ occupied by Rio Del Rey and $1100 \mathrm{~km}^{2}$ by Cameroon estuary. A study on the ecosystem services was carried out in the mangrove ecosystem of the Wouri estuary to estimate the financial stakes and pollution risks connected to the transport of persons and goods. A random sample of 27 canoes were taken in two main mangrove landing areas in the locality with 20 and 07 in Youpwè and Akwa-Nord respectively with observations using socio-economic data sheets. The results obtained show that the 
ecosystemic services constitute a means of livelihood for the riverine populations. The flux of persons and goods were important and amounts to a turnover of $12252600 \mathrm{~F} \mathrm{CFA} \mathrm{(24} 505$ USD)/canoe/year for Youpwè and 3896286 F CFA (7 792 USD)/canoe/year for Akwa-Nord respectively. However, the anthropogenic activities linked to this transport constitute threats to its perpetuity.

(c) 2015 International Formulae Group. All rights reserved.

Keywords: Mangrove, transport, ecosystemic services, financial stakes, threats.

\section{INTRODUCTION}

Le transport par voie d'eau en milieu rural est une activité qui permet le déplacement des marchandises et des passagers le long des voies navigables (Ruralwaterways, 2008). Cette navette est rendue possible grâce aux petites embarcations de fortune. Pour la plupart des personnes démunies, ce transport constitue le seul moyen de déplacement et d'accès aux services élémentaires (Ajonina, 2008). Les populations rurales des zones enclavées ont recours à ce transport comme mode d'accès et de déplacement pendant la majeure partie ou la moitié de l'année (Ruralwaterways, 2008). En Afrique en général, au Cameroun en particulier ce type de transport artisanal se pratique à travers les zones humides, dont l'une des principales est la mangrove (INSEE, 2013). Cette dernière fournit un large éventail des biens et services essentiels à la société. Selon la FAO (2006), les populations tant rurales qu'urbaines, y pratiquent la pêche (poissons et crevettes), la collecte d'autres produits halieutiques (moules, coquillages), le fumage du poisson, la chasse (gibier et mammifères marins), la coupe et la commercialisation du bois de palétuvier (bois d'œuvre et énergie). Les riverains pratiquent certaines activités telles que l'extraction du sable, la collecte des produits forestiers non ligneux (feuilles, tiges, écorce, fruits, miel), la fabrication, la réparation, l'entretien du matériel de pêche et de transport maritime (pirogues) (Din, 2001). Outre ces activités, les populations s'adonnent également au transport des biens et des personnes à travers la mangrove car cette dernière constitue le réseau des voies côtières privilégié pour ce type d'activité dans des zones où le transport routier est déficient (Nfotabong et al., 2009). Ce service de transport a pu être évalué et a été estimé à 1119060 F CFA/km/an selon les données de quelques 11 destinations de mangrove de Youpwè-Littoral-Cameroun, avec une moyenne de 20 personnes voyageant par pirogue (Ajonina et al., 2013).

Malgré l'importance du transport des biens et des personnes à travers la mangrove, très peu d'études ont déjà été menées sur la valeur économique de cette activité, raison pour laquelle les données portant sur la valeur économique liée à cette activité de transport à travers cet écosystème restent embryonnaires et éparses.

L'objectif principal de cette étude a été l'évaluation économique de l'activité de transport des personnes et des marchandises à travers la mangrove dans l'estuaire du Wouri. Spécifiquement, il s'agissait de caractériser l'activité de transport des personnes et des marchandises de la zone d'étude, d'évaluer le chiffre d'affaire du transport des personnes et des marchandises et d'estimer les impacts de ce transport sur la conservation et la gestion durable de la mangrove.

\section{MATERIEL ET METHODES \\ Généralités sur la zone d'étude}

Situé entre les latitudes $1^{\circ} 40^{\prime}$ et $13^{\circ} 05^{\prime}$ $\mathrm{N}$ et les longitudes $8^{\circ} 30^{\prime}$ et $16^{\circ} 10^{\prime} \mathrm{E}$, le Cameroun couvre une superficie de 475442 $\mathrm{km}^{2}$, et une grande partie de la côte camerounaise est occupée par la mangrove.

Les sites faisant l'objet de l'étude sont les zones de mangroves où s'effectue le transport artisanal fluvial dans la région du Littoral, département du Wouri, précisément dans les arrondissements de Douala $2^{\text {ème }}$ (marché de Youpwè) et de Douala $5^{\text {ème }}$ (marché d'Akwa-Nord), situés entre $03^{\circ} 91^{\prime}$ de latitude Nord et $09^{\circ} 42^{\prime}$ de longitude Est, en moyenne (Figure 1).

La ville de Douala, capitale économique du Cameroun, s'étale sur environ 18000 ha pour une population estimée à près de 2,5 millions d'habitants, dont les autochtones sont composés en majorité des 
«Bassa» et des «Duala». Cette ville regorge d'une multitude de religions qui cohabitent ensemble et dont les principales sont le catholicisme et le protestantisme, mais il est observé également une minorité musulmane (Régions du Cameroun, 2010).

La ville de Douala bénéficie d'un climat équatorial d'un type particulier dit «camerounien» (Bagnouls et Gaussen, 1957). Ce climat se caractérise par deux saisons avec une longue saison de pluies (au moins 9 mois), des précipitations abondantes (environ $4000 \mathrm{~mm}$ par an). La moyenne minimale de température à Douala pour 30 années (19611990) est de $22,6{ }^{\circ} \mathrm{C}$ en juillet et la moyenne maximale de $32,3{ }^{\circ} \mathrm{C}$ en février.

Pour l'année 2012, il est constaté que le mois de septembre est le plus pluvieux de l'année avec $1027 \mathrm{~mm}$ de précipitations alors que le mois de janvier est le plus sec avec seulement 24,1 mm. Quant aux températures, elles sont relativement élevées, avec une moyenne mensuelle de $27,7{ }^{\circ} \mathrm{C}$. Le mois de février étant le plus chaud, avec $29,1^{\circ} \mathrm{C}$ alors que le mois d'août est le plus froid, avec seulement $25,7^{\circ} \mathrm{C}$.

L'air est presque constamment saturé (99\% d'humidité relative en saison des pluies, mais $80 \%$ en saison sèche) (Toumba, 2012). L'humidité relative de l'air reste élevée toute l'année et voisine de 100\% (Din et al., 2008). L'altitude varie entre 1,6 et $39 \mathrm{~m}$ avec un vaste réseau hydrographique constitué par endroits de marécages et d'une nappe phréatique qui affleure dans les quartiers centraux (CUD, 2007). La ville est traversée dans sa région Nord-Ouest par le fleuve Wouri, qui est alimenté par de nombreuses rivières, (Din et al., 2008). Le relief est celui des terres basses côtières avec des formations sédimentaires (argiles, sables, grès, marnes,...) et une importante proportion de sols hydromorphes (CUD, 2005). Douala est une ville carrefour ayant reçue des cultures allogènes qui influencent fortement les cultures locales incluant les traditions et les pratiques médicinales traditionnelles.

Après prospection de la zone d'étude, le choix du site s'est porté sur les marchés de Youpwè et d'Akwa-Nord de la ville de Douala pour plusieurs raisons : l'intensité du trafic, car ces derniers constituent les zones de départ pour plusieurs destinations et la proximité des sites par rapport à la mangrove. En effet, ces deux sites sont proches des mangroves, où la circulation des pirogues se fait à travers les criques.

\section{Matériel}

La collecte et l'analyse des données ont été effectuées grâce à un matériel spécifique comprenant :

- un décamètre pour les mesures des dimensions des pirogues de transport;

- un appareil photo pour les illustrations du travail fait sur le terrain ;

- un ordinateur et des logiciels statistiques (Excel et SPSS) pour la saisie, le stockage, le traitement et l'analyse des données ;

- une fiche d'enquête socioéconomique sur laquelle a été porté un questionnaire.

\section{Méthodes}

La méthode d'échantillonnage s'est faite aléatoirement sur 27 pirogues dont 20 à Youpwè et 7 à Akwa-Nord. Grâce à des fiches d'enquêtes socio-économiques, les paramètres suivants ont été enregistrés auprès des acteurs de cette activité (transporteurs et passagers): la taille et le type de pirogues, le coût du transport des passagers et marchandises, le type et les différentes capacités des moteurs, la fréquence des voyages, les différents trajets effectués, les caractéristiques sociodémographiques des acteurs et les aspects négatifs liés à cette activité.

\section{Analyse statistique}

Ce calcul ne concerne que le chiffre d'affaire car c'est le seul paramètre qui nécessite l'utilisation d'une formule de calcul, ce chiffre d'affaire se donnera en fonction de la catégorie (taille) des pirogues et en fonction des sites, par la formule:

$\mathrm{CA}=\left[\left(\left(\mathrm{n}_{\mathrm{p}} \times \mathrm{p}_{\mathrm{p}}\right)+\mathrm{p}_{\mathrm{b}}\right) \times \mathrm{n}_{\mathrm{m}}\right]$

Avec : CA : Chiffre d'affaire; $p_{p}$ : Prix moyen du transport par passager; $\mathrm{p}_{\mathrm{b}}$ : Prix moyen de bagages; $\mathrm{n}_{\mathrm{p}}$ : Nombre moyen de passagers ; $\mathrm{n}_{\mathrm{m}}$ : Fréquence des voyages par pirogue.

Les autres paramètres ont été obtenus par analyse descriptive, notamment les caractéristiques socio-démographiques des personnes impliquées dans le transport (âge, nationalité, sexe, niveau d'étude), l'aspect logistique du transport (capacité des moteurs, 


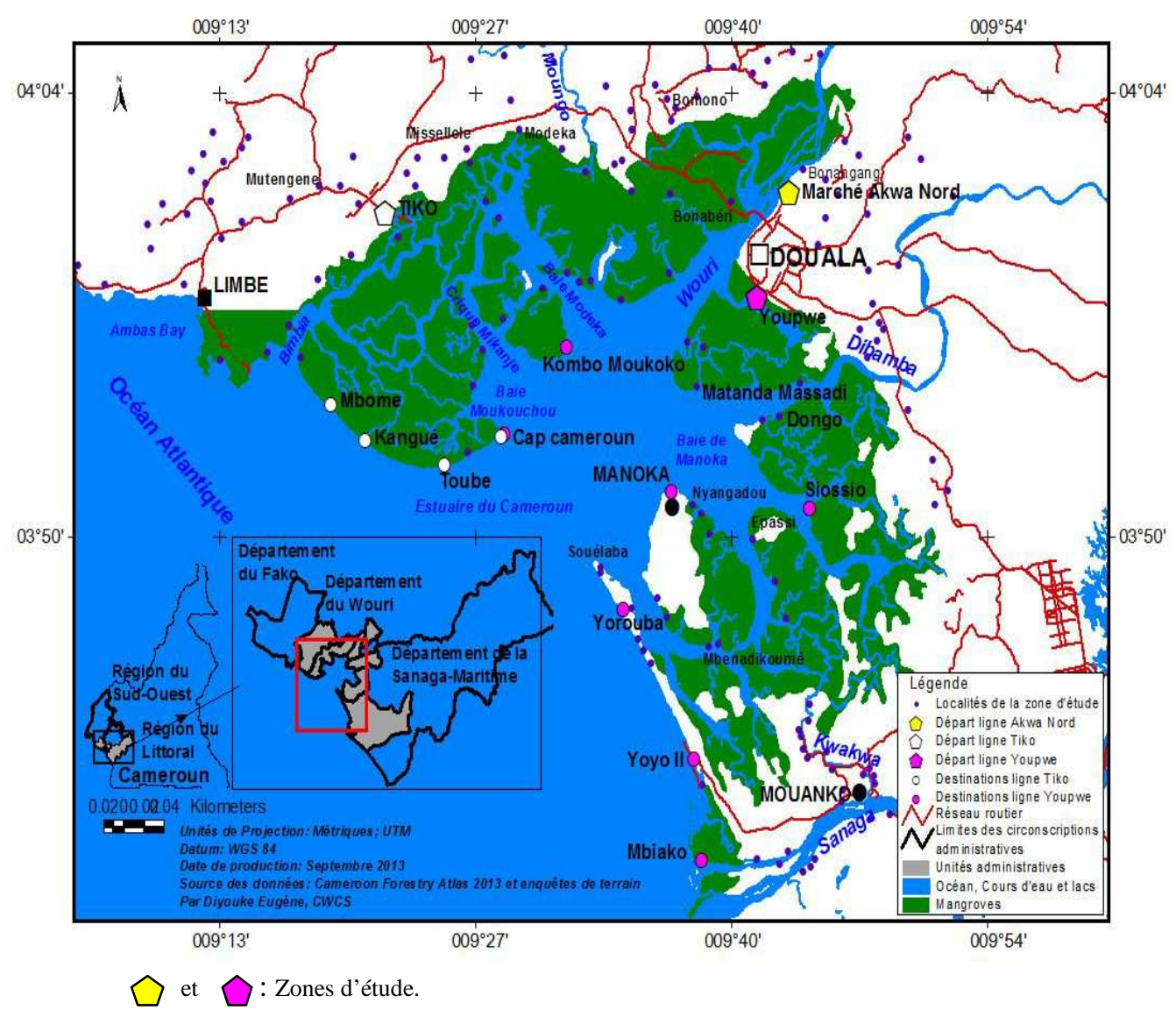

Figure 1 : Carte d'une partie de la mangrove de l'estuaire du Cameroun.

gilets de sauvetage, taille des pirogues, etc.), l'aspect négatif du transport (déversement des hydrocarbures, des produits chimiques, des déchets plastiques et même des excréments).

\section{RESULTATS}

Caractérisation de l'activité de transport des personnes et des marchandises dans les différents sites

\section{Aspect socio-démographique}

Les caractéristiques des personnes impliquées dans l'activité de transport ont été réparties en fonction du sexe, de l'âge, de la nationalité et du niveau d'étude (Figure 2).

A Akwa-Nord, il est observé deux fois plus d'hommes que de femmes $(66 \%$ contre $34 \%)$; tel n'est pas le cas à Youpwè où ces valeurs se rapprochent $(57 \%$ de sexe masculin contre $43 \%$ de sexe feminin). Les acteurs de transport intérrogés sont de trois nationalités : camerounaise, nigérianne et ghanéenne, à Youpwè mais sont tous camerounais, du côté d'Akwa-Nord. Ces acteurs sont majeurs, car $80 \%$ à $90 \%$ d'entre eux ont plus de 18 ans, respectivement à Youpwè et à Akwa-Nord. En ce qui concerne le niveau scolaire, seul $10 \%$ des acteurs de ce transport ont eu accès aux études supérieures du côté de Youpwè, 65\% aux études secondaires et $25 \%$ aux études primaires, contre $60 \%$ aux études secondaires et $40 \%$ aux études primaires, du côté d'AkwaNord.

\section{Etat des lieux de la logistique du transport} dans les différents sites

Toutes les pirogues sont en bois avec des moteurs hors-bords qui varient selon les 
capacités (Tableau 1). Pour le site de Youpwè, la capacité la plus élevée du moteur est située dans l'intervalle [30-40] chevaux pour $64 \%$ des cas ; tandis qu'à Akwa-Nord, les moteurs sont tous de faible capacité, [10-20] chevaux pour $100 \%$ de cas. Par ailleurs, la taille des pirogues varie selon les sites, seul Youpwè possède les trois catégories (petite 13\%; moyenne $54 \%$ et grande $33 \%$ ), alors qu'à Akwa-Nord, on observe l'absence des petites pirogues (moyennes pirogues $85 \%$ et grandes pirogues $15 \%$ ).

\section{Flux de transport dans les différents sites}

La variation des caractéristiques du flux est fonction des destinations dans les deux sites (Tableaux 2 et 3 ). C'est le cas à Kombo Moukoko où le nombre de passagers par voyage et par pirogue varie entre 18 et 40 pour une moyenne de 26 .

Evaluation du chiffre d'affaire dans les deux sites

Chiffre d'affaire d'Akwa-Nord

Le chiffre d'affaire annuel par pirogue de l'activité de transport d'Akwa-Nord est fonction de la catégorie des pirogues. En effet, il est plus important pour les grandes pirogues (4 692744 F CFA) par rapport aux moyennes pirogues (3 $591804 \mathrm{~F}$ CFA), avec une moyenne de $3896286 \mathrm{~F}$ CFA par pirogue.

\section{Chiffre d'affaire de Youpwè}

Dans le secteur de transport à Youpwè, on observe trois catégories de pirogues (petites, moyennes et grandes), et non deux comme à Akwa-Nord. La particularité ressort du chiffre d'affaire des petites pirogues (14 $448000 \mathrm{~F} \mathrm{CFA})$ qui semble plus important que celui des moyennes pirogues (12 252600 F CFA).

\section{Effet de l'activité de transport sur l'écosystème de Mangrove Déversement des hydrocarbures}

Le matériel utilisé par les transporteurs lors des voyages peut être défaillant. C'est ainsi que la totalité des personnes interrogées ont affirmé être souvent tombées en panne mécanique ou sèche lors des voyages. Parmi ces dernières, près de $73 \%$ à Youpwè et $67 \%$ à Akwa-Nord ont affirmé qu'elles déversent de l'huile de moteur et du carburant dans l'eau pendant la manipulation, lors du dépannage de leur moteur (Figure 3).

\section{Déversement des déchets plastiques}

Le manque d'accessoires tels que les poubelles dans les pirogues artisanales de transport mêlés aux comportements immoraux des passagers constituent l'un des facteurs de pollution des mangroves. Certains passagers après avoir consommé un produit, pour la plupart alimentaire, jettent l'emballage dans l'eau et ces déchets vont échouer dans la mangrove. $100 \%$ à Youpwé et $37 \%$ à AkwaNord de personnes interrogées ont affirmé avoir commis cet acte.

\section{Probabilité de déversement des produits chimiques et autres types de produits}

Pendant les voyages, les transporteurs rencontrent souvent des difficultés liées aux collisions entre embarcations, aux collisions avec des bancs de sable, aux vents violents engendrant de grandes vagues pouvant faire chavirer les pirogues. Près de $70 \%$ de transporteurs à Youpwè et $25 \%$ à Akwa-Nord ont affirmé avoir eu des collisions avec d'autres embarcations et près de $80 \%$ de transporteurs à Youpwè et $85 \%$ à Akwa-Nord ont affirmé avoir eu des collisions avec des bancs de sable ou ont eu à affronter des vagues. Les bagages tombés dans l'eau peuvent constituer un danger pour l'écosystème de mangrove s'ils contiennent des produits chimiques tels que : les produits pharmaceutiques (80\% Youpwè et $57 \%$ Akwa-Nord), les pesticides et herbicides agricoles (62\% Youpwè et $100 \%$ Akwa-Nord) et cosmétiques (95\% Youpwè et $71 \%$ AkwaNord) (Figure 4).

\section{Déversement de la matière fécale}

Le manque de toilettes dans ces pirogues amène les passagers à se mettre à l'aise à l'air libre pendant le voyage. Ainsi, sur près de 37 transporteurs interrogés, $75 \%$ à Akwa-Nord et $100 \%$ à Youpwè ont affirmé que leurs passagers se mettent souvent à l'aise dans l'eau pendant le voyage (Figure 5). 


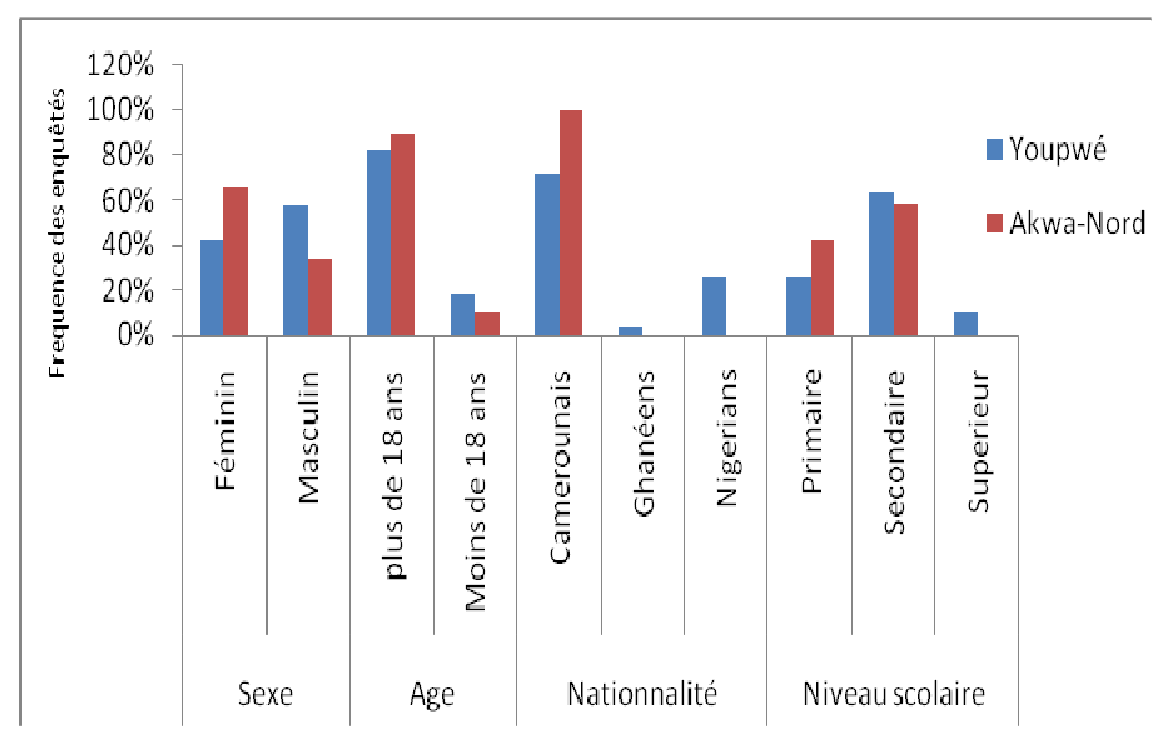

Figure 2: Caractéristiques démographiques des populations impliquées dans le transport dans les deux sites.

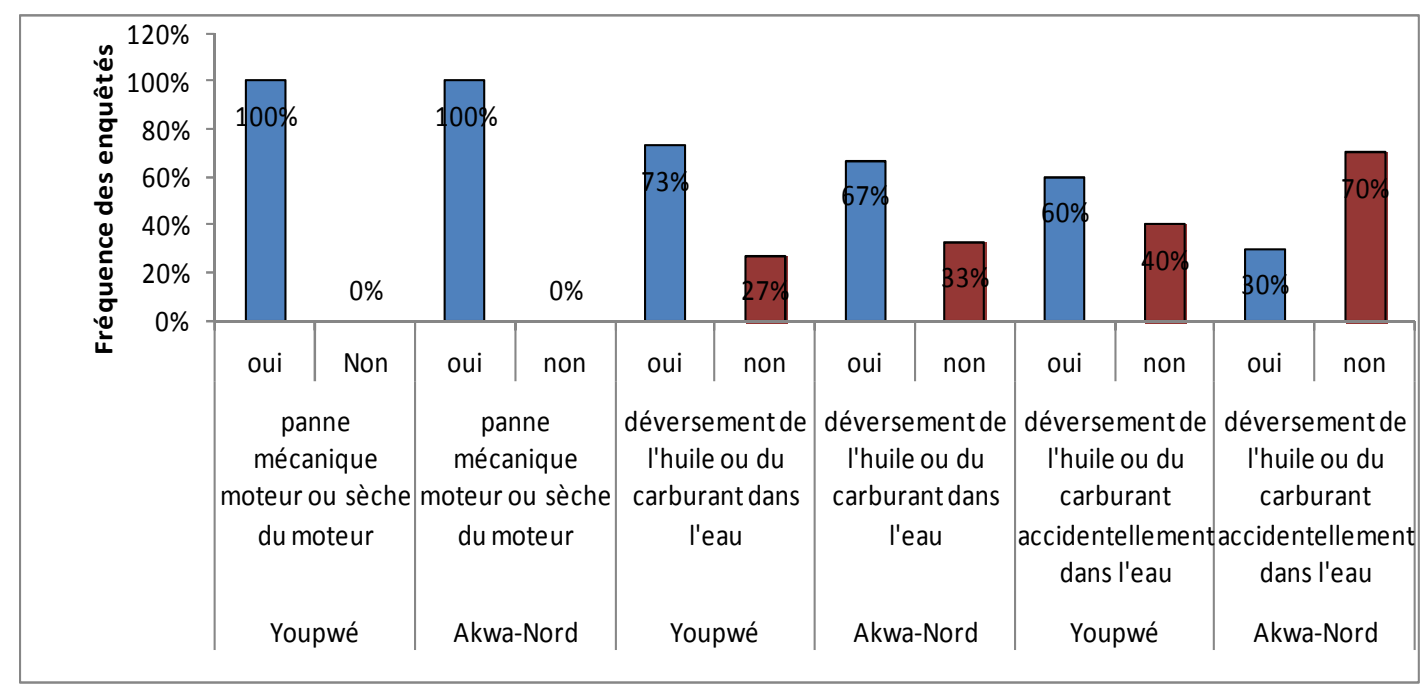

Figure 3 : Fréquence de différentes pannes de moteur et déversement du carburant dans les différents sites. 


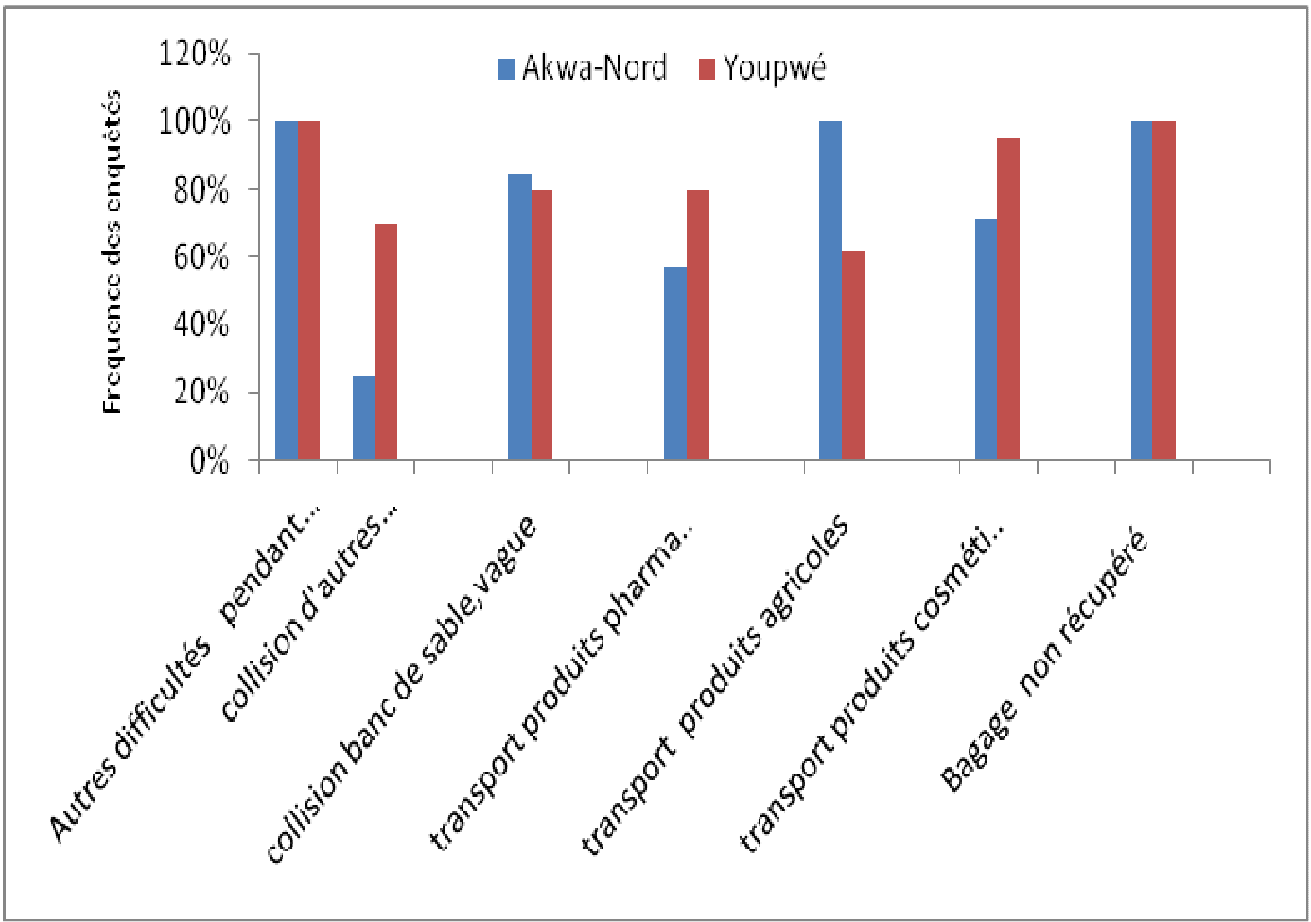

Figure 4 : Probabilité de déversement des produits chimiques et autres types de produits dans l'eau lors du transport.

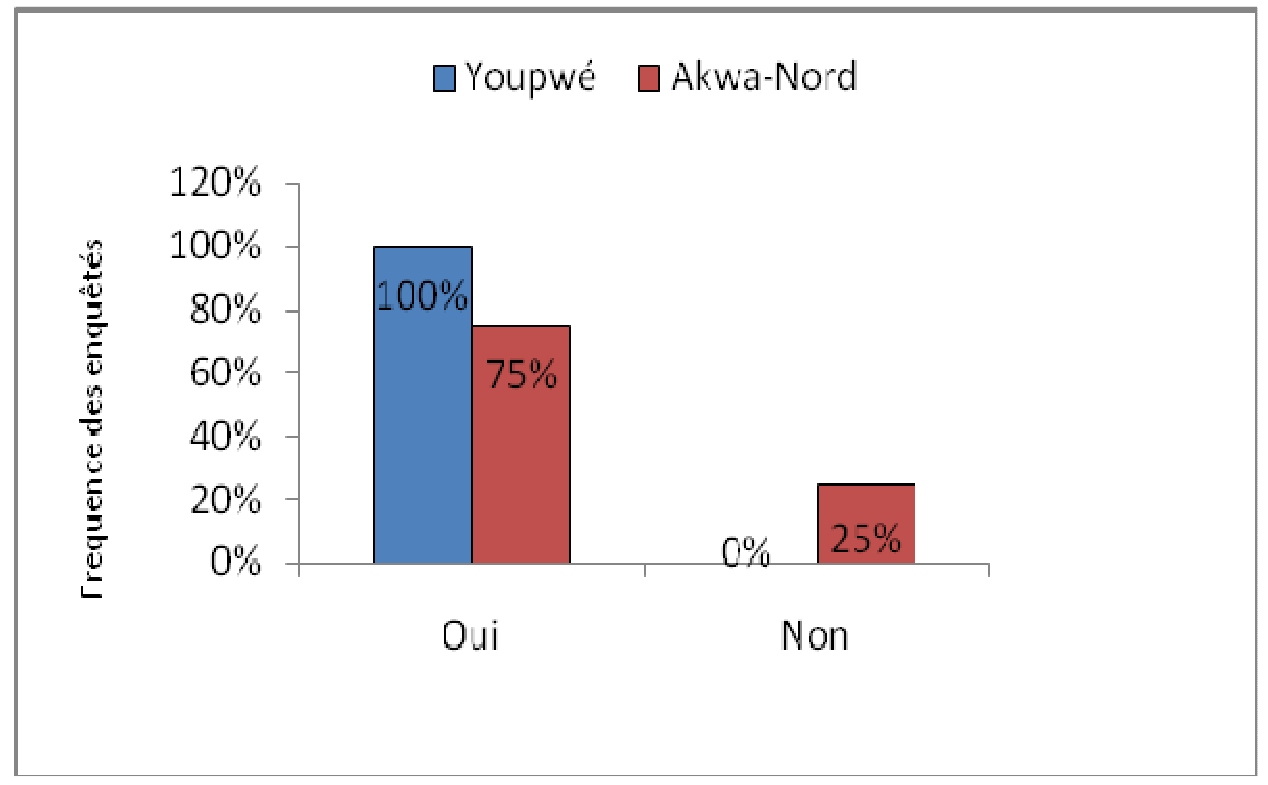

Figure 5 : Fréquence des personnes se mettant souvent à l'aise dans l'eau. 
Tableau 1 : Logistiques de transport des personnes et marchandises dans les deux sites.

\begin{tabular}{|c|c|c|c|c|c|c|c|c|c|c|c|c|c|}
\hline & \multicolumn{3}{|c|}{ Taille des pirogues } & \multirow{2}{*}{$\begin{array}{c}\begin{array}{c}\text { Type de } \\
\text { pirogue } \\
\text { (matériel) }\end{array} \\
\text { Planche }\end{array}$} & \multirow{2}{*}{$\begin{array}{c}\begin{array}{c}\text { Gilet de } \\
\text { sauvetage }\end{array} \\
\text { Présent }\end{array}$} & \multicolumn{3}{|c|}{ Capacité du moteur (CV) } & \multirow{2}{*}{$\begin{array}{c}\text { Type de } \\
\text { moteur } \\
\text { Hors- } \\
\text { bord }\end{array}$} & \multirow{2}{*}{$\begin{array}{l}\begin{array}{c}\text { Lampe } \\
\text { torche }\end{array} \\
\text { Présent }\end{array}$} & \multirow{2}{*}{$\begin{array}{c}\begin{array}{c}\text { Ancre avec } \\
\text { longueur de la } \\
\text { corde }>\mathbf{3 0 ~} \mathbf{~ m}\end{array} \\
\text { Présent }\end{array}$} & \multirow{2}{*}{$\begin{array}{c}\text { Extincteur } \\
\text { Absent }\end{array}$} & \multirow{2}{*}{$\begin{array}{c}\begin{array}{c}\text { Permis ou } \\
\text { capacité et } \\
\text { certificat d } \\
\text { navigabilit }\end{array} \\
\text { Présent }\end{array}$} \\
\hline & Petite & Moy. & Grande & & & {$[10-20]$} & {$[20-30]$} & {$[30-40]$} & & & & & \\
\hline Youpwè & $13 \%$ & $54 \%$ & $33 \%$ & $100 \%$ & $100 \%$ & $0 \%$ & $36 \%$ & $64 \%$ & $100 \%$ & $100 \%$ & $100 \%$ & $100 \%$ & $100 \%$ \\
\hline Akwa-Nord & $0 \%$ & $85 \%$ & $15 \%$ & $100 \%$ & $0 \%$ & $100 \%$ & $0 \%$ & $0 \%$ & $100 \%$ & $100 \%$ & $100 \%$ & $100 \%$ & $100 \%$ \\
\hline
\end{tabular}


G.N. AJONINA et al. / Int. J. Biol. Chem. Sci. 9(4): 1851-1862, 2015

Tableau 2: Variation des paramètres des flux de transport en fonction des destinations des pirogues du site de Youpwé.

\begin{tabular}{|c|c|c|c|c|c|}
\hline Paramètre des flux & Destinations & Moyenne & Erreur standard & Minimum & Maximum \\
\hline \multirow{7}{*}{ Nombre de passagers /voyage/ pirogue } & Cap-Cameroun & 19 & 1 & 8 & 35 \\
\hline & Kombo Moukoko & 26 & 2 & 18 & 40 \\
\hline & Siosio & 16 & 5 & 10 & 25 \\
\hline & Yorouba & 20 & 1 & 15 & 26 \\
\hline & Yoyo & 18 & 1 & 11 & 23 \\
\hline & Manoka & 14 & 6 & 5 & 25 \\
\hline & Total & 20 & 1 & 5 & 40 \\
\hline \multirow{7}{*}{ Prix des bagages/voyage/ pirogue (F CFA) } & Cap-Cameroun & 32539 & 2829 & 12000 & 70000 \\
\hline & Kombo Moukoko & 17471 & 3192 & 3000 & 40000 \\
\hline & Siosio & 35667 & 7446 & 25000 & 50000 \\
\hline & Yorouba & 33250 & 3442 & 17000 & 45000 \\
\hline & Yoyo & 25600 & 3033 & 18000 & 45000 \\
\hline & Manoka & 34950 & 2844 & 30000 & 39850 \\
\hline & Total & 27826 & 1799 & 3000 & 70000 \\
\hline \multirow{7}{*}{$\begin{array}{l}\text { Tarif de passager/ voyage/pirogue } \\
\text { (F CFA) }\end{array}$} & Cap-Cameroun & 1480 & 19 & 1000 & 1500 \\
\hline & Kombo Moukoko & 1000 & 0 & 1000 & 1000 \\
\hline & Siosio & 1500 & 0 & 1500 & 1500 \\
\hline & Yorouba & 1938 & 63 & 1500 & 2000 \\
\hline & Yoyo & 1500 & 0 & 1500 & 1500 \\
\hline & Manoka & 1500 & 0 & 1500 & 1500 \\
\hline & Total & 1500 & 47 & 1000 & 2500 \\
\hline \multirow{7}{*}{ Nombre de voyages/ pirogue/mois } & Cap-Cameroun & 27 & 1 & 14 & 28 \\
\hline & Kombo Moukoko & 7 & 0 & 7 & 7 \\
\hline & Siosio & 13 & 1 & 12 & 14 \\
\hline & Yorouba & 14 & 0 & 14 & 14 \\
\hline & Yoyo & 15 & 1 & 14 & 28 \\
\hline & Manoka & 14 & 0 & 14 & 14 \\
\hline & Total & 18 & 1 & 7 & 28 \\
\hline
\end{tabular}


G.N. AJONINA et al. / Int. J. Biol. Chem. Sci. 9(4): 1851-1862, 2015

Tableau 3: Variation des paramètres des flux de transport en fonction des destinations des pirogues du site d'Akwa-Nord.

\begin{tabular}{llcccc}
\hline Paramètre des flux & Destinations & Moyenne & Erreur standard & Minimum & Maximum \\
\hline \multirow{2}{*}{ Nombre de passagers/ } & Bodiman & 13 & 1 & 8 & 17 \\
voyage/pirogue & Ewodi & 14 & 1 & 9 & 23 \\
& Total & 13 & 1 & 8 & 23 \\
\hline \multirow{2}{*}{ Prix d'un passage/ } & Bodiman & 1500 & 0 & 1500 & 1500 \\
voyage/pirogue (F CFA) & Ewodi & 1000 & 0 & 1000 & 1000 \\
& Total & 1481 & 19 & 1000 & 1500 \\
\hline \multirow{2}{*}{ Prix des bagages/ } & Bodiman & 12891 & 3118 & 0 & 38500 \\
voyage/pirogue (F CFA) & Ewodi & 12862 & 3576 & 0 & 37500 \\
& Total & 12879 & 2320 & 0 & 38500 \\
\hline \multirow{2}{*}{ Nombre de } & Bodiman & 11 & 1 & 8 & 17 \\
voyages/pirogue/mois & Ewodi & 11 & 0 & 8 & 16 \\
& Total & 11 & 1 & 8 & 17 \\
\hline
\end{tabular}




\section{DISCUSSION}

L'écosystème mangrove est le théâtre d'intenses activités économiques menées par une population riveraine en pleine croissance et estimée à environ 500000 habitants (Ajonina et al., 2013). Cet écosystème sain fournit à la société un large éventail des biens et services essentiels (produits de pêche, bois d'énergie, transport des marchandises et des personnes).

Les flux de transport ont varié non seulement en fonction des destinations mais aussi en fonction de la quantité des marchandises et du nombre de passagers. Ces flux ont révélé dans le site de Youpwè, une moyenne de 20 passagers par pirogue, valeur proche de 20,5 obtenue par Ajonina et al. (2013). Par contre, dans le site d'Akwa-Nord, concernant le prix des bagages par voyage et par pirogue, cette valeur moyenne a été estimée à $12879 \pm 2320 \mathrm{~F}$ CFA, valeur éloignée de 925 F CFA obtenue également par Ajonina et al. (2013). La variation de la logistique est fonction des sites, elle peut être due, soit à l'intensité du trafic dans les différents sites, soit aux moyens dont disposent les propriétaires dans l'acquisition des catégories de pirogues. Cette différence catégorielle s'explique par la taille des pirogues mais aussi par la fréquence des voyages (les petites pirogues qui se remplissent vite, font plus de voyages).

Les individus de nationalités diverses se déplacent sans distinction de sexe sur une même pirogue comme dans les régions d'Asie (Ruralwayswater, 2008). La pratique de cette activité est l'apanage de plusieurs nationalités: camerounaise, nigérianne, béninoise et ghannéenne.

La différence dans le chiffre d'affaire, à Youpwè par exemple, 21.900.000 F CFA selon Ajonina et al. (2013) contre 12.252.600 F CFA, s'explique par certains facteurs caractérisant cette évaluation économique, notamment le prix moyen des passagers estimé à $1885 \mathrm{~F}$ CFA par Ajonina et al. (2013) au lieu de 1500 F CFA pour notre étude, mais aussi par le nombre de destinations : onze contre cinq observées pour nos deux stations. Par contre, dans le site
d'Akwa-Nord, il a été noté une valeur moyenne de 3896286 F CFA de chiffre d'affaire par pirogue inférieure à celle évaluée à Youpwè, pouvant s'expliquer par l'intensité du trafic plus important dans ce dernier site.

Sous l'effet cumulé de diverses actions anthropiques, l'écosystème de mangrove subit des pollutions diverses de façon continue. Ces pollutions sont donc considérées comme des facteurs contribuant à la disparition faunique et floristique des espèces de mangrove comme le signalent Baltzer et al. (1995), Din et al. (2001), Waffo (2009) et Ajonina et al. (2013) pour certaines plantes médicinales et espèces de poissons à Yoyo-Mouanko.

\section{Conclusion}

Au terme de cette étude, il ressort que la fréquence des critères démographiques des individus impliqués dans le transport a varié selon les sites. Les individus se déplacent principalement pour le commerce des produits halieutiques et vivriers, au moyen des pirogues en bois propulsées par des moteurs hors-bords dont la capacité est fonction de la densité du trafic. Youpwè a été considéré comme étant le site ayant le plus grand trafic car il y est observé une fréquence plus élevée de passagers et un chiffre d'affaire plus important (12 252600 F CFA). Malgré l'importance économique de cette activité, il ressort qu'elle peut contribuer également à la pollution de l'écosystème de mangrove.

\section{CONFLIT D'INTERET}

Les auteurs déclarent qu'il n'y a aucun conflit d'intérêt.

\section{CONTRIBUTIONS DES AUTEURS}

GNA, SDD et RES ont conçu le thème et superviser le travail. NEN a participé aux corrections de la partie économique de ce travail. YG-M a participé à la collecte des données, ainsi qu'à la traduction des documents utilisés. AN a été de toutes les campagnes de terrain liées à ce travail. Tous les auteurs ont participé à la rédaction du manuscrit. 


\section{REMERCIEMENTS}

Les auteurs remercient tous ceux qui ont facilité cette étude, surtout les autorités maritimes de Douala, mais aussi Monsieur Diyouke Eugène de la Cameroon Wildlife Conservation Society (CWCS) pour la production de la carte.

\section{REFERENCES}

Ajonina G, Ayissi I. 2012. Linking river hydrodynamics and sedimentology to water bird numbers from longterm monthly monitoring of water birds within the lower Sanaga Basin, Cameroon. Abstracts, 13 Pan African Ornithological Congress, Arusha, Tanzania, p. 7.

Ajonina G, Kemajou J, Bitchick AC, Nguekambe. 2013. Rapport technique sur l'évaluation chiffrée de la dégradation d'une partie de la mangrove du littoral: OPED 2013, p. 103.

Baltzer F, Rudant JP, Kuete M, Bilong P, Monteillet J, Abossolo, Amougou A, Din, N, Tonye E, Abata T. 1995. Etude des mangroves de Douala (Cameroun) par imagerie radar et contrôle de terrain. Rapport, Orsay, p. 22.

CUD (Communauté Urbaine de Douala). 2007. Service de cartographie et de plans urbains, CUD.

CUD (Communauté Urbaine de Douala). 2005. Schéma directeur d'aménagement et d'urbanisme de Douala; Projet de Développement Urbain (PDU), drainage principal de Douala, p. 15.

Din N, Priso RJ, Dibong SD, Amougou A. 2001. Identification des principales causes de dégradation des mangroves dans l'estuaire du Cameroun. Sci. Technol. Dev., 8(1): 1-7.

Din N, Senger P, Priso JR, Dibong SD, Amougou A. 2008. Logging activities in mangroves forests. A case study of Douala Cameroon. Africa Journal in Environmental Science and Technology, 2: $22-30$.

FAO (Organisation des Nations Unies pour l'Alimentation et l'Agriculture). 2006.
Politique et stratégie pour la gestion durable des écosystèmes de mangroves du Cameroun, Gestion participative et conservation de la diversité biologique des mangroves TCP/CMRJ2908. p. 44.

INSEE (Institut Nationale de Statistique et des Etudes Economiques). 2013. Définitions et méthodes de transport 2013, p. 82.

Nfotabong AA, Din N, Longonje SN, Koedam N, Dahdouh-Guebas F. 2009. Commercial activities and subsistence utilization of mangrove forests around the Wouri estuary and the Douala-Edea reserve (Cameroon). Journal of Ethnobiology and Ethnomedicine, 5: 35.

Ruralwaterways, 2008. Qu'est-ce que le transport par voie d'eau en milieu rural? p. 22.

Toumba G. 2012. Rapport d'activité du Ministère de l'élevage; des Pêches et des Industries Animales du Littoral. p. 128.

Ajonina G. 2008. Inventory and modeling mangrove forest stand dynamics following different levels of wood exploitation pressures in the DoualaEdea Atlantic coast of Cameroon, Central Africa. Mitteilungen der Abteilungen für Forstliche Biometrie, Albert-Ludwigs-Universität Freiburg. 2008-2. p. 215.

Bagnouls F, Gaussen H. 1957. Les climats biologiques et leurs significations ; 193220.

Din Ndongo. 2001. Mangroves du Cameroun. Statut écologique et perspectives de gestion durable. Thèse de Doctorat d'Etat ès Sciences. Option écologie végétale. Faculté des Sciences de l'Université de Yaoundé I, Yaoundé, p. 246.

Waffo Ulrich. 2009. Menaces sur la mangrove au Cameroun. Maîtrise en Géographie. Université de Yaoundé I. p. 21.

Régions du Cameroun. 2010. Le Cameroun en bref 2010. http://www.planetere. org/bulletin/ 2010/ pdf-doc/Regionsdu-Cameroun. PDF. 